\title{
ON UNIVERSAL SPACES FOR THE CLASS OF BANACH SPACES WHOSE DUAL BALLS ARE UNIFORM EBERLEIN COMPACTS
}

\author{
CHRISTINA BRECH AND PIOTR KOSZMIDER
}

(Communicated by Thomas Schlumprecht)

\begin{abstract}
For $\kappa$ being the first uncountable cardinal $\omega_{1}$ or $\kappa$ being the cardinality of the continuum $\mathfrak{c}$, we prove that it is consistent that there is no Banach space of density $\kappa$ in which it is possible to isomorphically embed every Banach space of the same density which has a uniformly Gâteaux differentiable renorming or, equivalently, whose dual unit ball with the weak* topology is a subspace of a Hilbert space (a uniform Eberlein compact space). This complements a consequence of results of M. Bell and of M. Fabian, G. Godefroy, and V. Zizler which says that assuming the continuum hypothesis, there is a universal space for all Banach spaces of density $\kappa=\mathfrak{c}=\omega_{1}$ that have a uniformly Gâteaux differentiable renorming. Our result implies, in particular, that $\beta \mathbb{N} \backslash \mathbb{N}$ may not map continuously onto a compact subset of a Hilbert space with the weak topology of density $\kappa=\omega_{1}$ or $\kappa=\mathfrak{c}$ and that a $C(K)$ space for some uniform Eberlein compact space $K$ may not embed isomorphically into $\ell_{\infty} / c_{0}$.
\end{abstract}

\section{INTRODUCTION}

A classical result of Banach and Mazur states that every separable Banach space can be isometrically embedded into the Banach space $C([0,1])$. In this paper we deal with the problem of embedding nonseparable Banach spaces of a given class into a single nonseparable space. We are interested in two uncountable densities, $\omega_{1}$ and $\mathfrak{c}$. Since a continuous onto map $\phi: K \rightarrow L$ (for $K, L$ compact Hausdorff spaces) gives an isometric embedding $T(f)=f \circ \phi$ of the Banach space $C(L)$ into $C(K)$, we have the following strictly related notions of universality:

Definition 1.1. Let $\mathcal{X}$ be a class of Banach spaces. We say that $X \in \mathcal{X}$ is (isometrically), a universal space for the class $\mathcal{X}$ if for every $Y \in \mathcal{X}$ there is an (isometric) isomorphism onto its range $T: Y \rightarrow X$.

Let $\mathcal{K}$ be a class of compact Hausdorff spaces. We say that $K \in \mathcal{K}$ is a universal space for the class $\mathcal{K}$ if for every $L \in \mathcal{K}$ there is a continuous onto map $T: K \rightarrow L$.

The following proposition shows that universal compact Hausdorff spaces and universal Banach spaces are closely related.

Received by the editors March 14, 2011 and, in revised form, August 5, 2011.

2010 Mathematics Subject Classification. Primary 46B26; Secondary 03E35, 46B03.

The first author was partially supported by FAPESP (2010/12638-1) and Pró-reitoria de Pesquisa USP (10.1.24497.1.2).

The second author was partially supported by Polish Ministry of Science and Higher Education research grant N N201 386234. 
Proposition 1.2. Suppose $\mathcal{K}$ is a class of compact Hausdorff spaces and $\mathcal{X}$ is a class of Banach spaces such that for each $K \in \mathcal{K}, C(K) \in \mathcal{X}$ and for each $X \in \mathcal{X}$, the dual unit ball $B_{X^{*}}$ with the weak* topology is in $\mathcal{K}$. If $K$ is a universal compact space for $\mathcal{K}$, then $C(K)$ is an isometrically universal Banach space for $\mathcal{X}$.

Proof. Let $X \in \mathcal{X}$. It is well known that $X$ isometrically embeds into $C\left(B_{X^{*}}\right)$. By the hypothesis, $B_{X^{*}} \in \mathcal{K}$, and so, $K$ continuously maps onto $B_{X^{*}}$, which means that $C\left(B_{X^{*}}\right)$ isometrically embeds into $C(K)$. Composing this isometric embedding with the isometric embedding of $X$ into $C\left(B_{X^{*}}\right)$, we obtain the desired embedding of $X$ into $C(K)$.

Let $\kappa$ be an infinite cardinal. Examples of pairs of classes $\mathcal{K}$ and $\mathcal{X}$ satisfying the hypothesis of the above proposition are:

- the class of all compact spaces of weight $\leq \kappa$ and the class of all Banach spaces of density $\leq \kappa$,

- the class of all Eberlein compact spaces (compact subsets of Banach spaces with the weak topology) of weight $\leq \kappa$ and the class of all weakly compactly generated Banach spaces (WCG) of density $\leq \kappa$,

- the class of all uniform Eberlein compact spaces (compact subsets of Hilbert spaces with the weak topology) of weight $\leq \kappa$ and the class of all Banach spaces which have a uniformly Gâteaux differentiable renorming (UG) of density $\leq \kappa$.

The nonexistence of a universal Banach space implies, by the above, the nonexistence of a corresponding universal compact space, but the opposite direction is not immediate. There are some reasons for this. First, $C(K)$ may be universal without $K$ being universal, as in the case of $K=[0,1]$ for the class of separable Banach spaces and metrizable compact spaces (however $C(K)$ is isomorphic to $C(\Delta)$, where $\Delta$ is the Cantor set, which is universal for metrizable compact spaces). Secondly, there are universal Banach spaces which are not isometrically universal. For example, consider a strictly convex renorming $(\|x+y\|=2$ for $\|x\|=\|y\|=1$ implies $x=y$ ) of $C([0,1]$ ) (which exists by Theorem 9 of [5] ) and notice that this space continues to be an isomorphically universal space but cannot isometrically include spaces whose norm is not strictly convex.

In the case of nonseparable Banach spaces we encounter mainly negative results concerning universality or results showing that to obtain a universal space one has to assume additional set-theoretic axioms. For example, it was proved in [2] that there are no universal WCG Banach spaces of density $\omega_{1}$ or $\mathfrak{c}$ nor universal Eberlein compact spaces of weight $\omega_{1}$ or $\mathfrak{c}$.

Assuming the continuum hypothesis, the compact space $\beta \mathbb{N} \backslash \mathbb{N}$ is a universal compact space of weight $\mathfrak{c}$ and by the above proposition $\ell_{\infty} / c_{0} \equiv C(\beta \mathbb{N} \backslash \mathbb{N})$ is an isometrically universal Banach space for the class of spaces of density $\mathfrak{c}=\omega_{1}$. However, it was shown in [7] that it is consistent that there is no isomorphically universal space of density $\mathfrak{c}$. K. Thompson and the second author noted that a version of the proof from [7] gives the consistency of the nonexistence of a universal Banach space of density $\omega_{1}$.

In 3] M. Bell showed that assuming $\kappa^{\omega}=\kappa$ there is a universal uniform Eberlein compact space of weight $\kappa$. M. Fabian, G. Godefroy and V. Zizler showed that the class of Banach spaces satisfying the hypothesis of Proposition 1.2 corresponding to the class of uniform Eberlein compact spaces is the class of UG Banach spaces. 
So, these two results imply by Proposition 1.2 that there are universal UG Banach spaces of density $\mathfrak{c}=\omega_{1}$. M. Bell also showed in 3] that it is consistent that there is no universal Eberlein compact space of density $\omega_{1}$. The main result of this paper is to complement the above result by its Banach space version. Actually the full result is considerably stronger:

Theorem 1.3. For $\kappa=\omega_{1}$ and for $\kappa=\mathfrak{c}$, it is consistent that there is no Banach space $X$ of density $\kappa$ such that every $U G$ Banach space of density $\kappa$ embeds into $X$. In particular, there is no universal Banach space for the class of UG Banach spaces of density $\kappa$.

As in the case of the class of separable spaces, the notions of universality and isometrical universality do not coincide for the class of UG spaces: a universal UG Banach space may not be an isometrically universal UG Banach space, and this follows from the fact that WCG Banach spaces can be renormed to be strictly convex (see [1]).

The above theorem implies in particular that, consistently, $\ell_{\infty} / c_{0}$ does not contain an isomorphic copy of some UG Banach spaces of density $\mathfrak{c}$. This is related to some recent results of M. Džamonja and L. Soukup [8] as well as to recent results of S. Todorcevic ([13]) concerning the consistent existence of $C(K) \mathrm{s}$ for $K$ Corson compact which do not embed into $\ell_{\infty} / c_{0}$. Also, the arguments as in the proof of Proposition 1.2 give that $\beta \mathbb{N} \backslash \mathbb{N}$ cannot be mapped onto some compact subset of a Hilbert space with density $K$ with the weak topology.

The notation is fairly standard, the Banach spaces terminology follows [9] and the set-theoretic terminology follows [11. Given a function $f$, we denote by $G_{f}$ the graph of $f . F n_{<\omega}(n, D)$ stands for all finite partial functions from $\{0, \ldots, n-1\}$ into $D$ and $F n_{<\omega}(\omega, D)$ (resp. $F n_{\leq \omega}(\omega, D)$ ) for all finite (resp. countable) partial functions from $\omega$ into $D$. If $X$ is a set, then $[X]^{1}$ denotes the collection of oneelement subsets of $X$. If $\mathcal{A}$ is a Boolean algebra and $a \in \mathcal{A}$, then $[a]$ denotes the basic clopen set of the Stone space of $\mathcal{A}$ determined by $a$, that is, the set of all ultrafilters of $\mathcal{A}$ containing $a$. A partial order has precaliber $\omega_{1}$ if and only if every uncountable subset of it includes an uncountable subset where every finite subset has a lower bound.

Definition 1.4. A Boolean algebra $\mathcal{A}$ is a c-algebra if it is generated by elements $\left\{A_{\xi, i}: \xi<\kappa, i<\omega\right\}$ of countably many pairwise disjoint antichains $\mathcal{A}_{i}=\left\{A_{\xi, i}\right.$ : $\xi<\kappa\}$ (collections of pairwise disjoint elements of $\mathcal{A}$ ) such that

(c) $A_{\xi_{1}, i_{1}} \vee \ldots \vee A_{\xi_{m}, i_{m}} \neq 1_{\mathcal{A}}$ for any distinct pairs $\left(i_{1}, \xi_{1}\right), \ldots,\left(i_{m}, \xi_{m}\right) \in \omega \times \kappa$.

We use a fact proved in [3] (Theorems 2.1 and 2.2) based on the results of 6 ] that the Stone space of a c-algebra $\mathcal{A}$ is a uniform Eberlein compact space. The link between uniform Eberlein compact spaces and UG Banach spaces is based on the following:

Theorem 1.5 (Theorem 2, [10]).

(1) A Banach space $X$ admits an equivalent uniformly Gâteaux differentiable norm if and only if the dual unit ball $B_{X^{*}}$ equipped with the weak* topology is a uniform Eberlein compact space.

(2) A compact space $K$ is a uniform Eberlein compact space if and only if $C(K)$ admits an equivalent uniformly Gâteaux differentiable norm if and only if 
there is a Hilbert space $\mathcal{H}$ and a bounded linear operator from $\mathcal{H}$ onto a dense set in $C(K)$.

The main idea of the proofs is to construct a large family of generic c-algebras and prove that given a UG Banach space $X$ one of the algebras is sufficiently generic over $X$ to prevent a possibility of an isomorphic embedding of the Banach space $C(K)$ where $K$ is the Stone space of the algebra. The c-algebras are constructed by partial order of approximations (see Definitions 2.1 and 3.1) and the method of forcing is used to make conclusions about their consistent existence (see [1]).

\section{Density $\omega_{1}$}

This section is devoted to the proof of the main result (Theorem 1.3) for the case $\kappa=\omega_{1}$. The following partial order will approximate the generic c-algebras of cardinality $\omega_{1}$, one of which will induce a Banach space UG without an isomorphic embedding into a given Banach space UG.

Definition 2.1. $\mathbb{P}$ is the forcing notion consisting of conditions $p=\left(n_{p}, D_{p}, F_{p}\right)$, where $n_{p} \in \omega, D_{p} \in\left[\omega_{1}\right]^{<\omega}$ and $F_{p} \subseteq F n_{<\omega}\left(n_{p}, D_{p}\right)$ are such that $\left|F_{p}\right|<\omega$ and $\left[n_{p} \times D_{p}\right]^{1} \subseteq F_{p}$, ordered by $p \leq q$ if $n_{p} \geq n_{q}, D_{p} \supseteq D_{q}, F_{p} \supseteq F_{q}$ and

(P) given $f \in F_{p}$, there is $g \in F_{q}$ such that $G_{f} \cap\left(n_{q} \times D_{q}\right) \subseteq G_{g}$.

Given a model $V$ and a $\mathbb{P}$-generic filter $G$ over $V$, for each $\xi \in \omega_{1}$ and each $i \in \omega$, we define in $V[G]$ the following set:

$$
A_{\xi, i}=\left\{f \in F n_{<\omega}\left(\omega, \omega_{1}\right): \exists p \in G \text { such that } f \in F_{p} \text { and } f(i)=\xi\right\} .
$$

Let $\mathcal{B}$ be the subalgebra of the Boolean algebra $\wp\left(F n_{<\omega}\left(\omega, \omega_{1}\right)\right)$ generated by the sets $\left\{A_{\xi, i}:(i, \xi) \in \omega \times \omega_{1}\right\}$.

Lemma 2.2. For every $\xi \in \omega_{1}, i \in \omega$ and every $q \in \mathbb{P}$ there is $p \leq q$ such that $\xi \in D_{p}$ and $i<n_{p}$.

Proof. Let $n_{p}=\max \left(i+1, n_{q}\right)$ and $D_{q}=D_{p} \cup\{\xi\}, F_{p}=F_{q} \cup\left[n_{p} \times D_{p}\right]^{1}$. Clearly $p \in \mathbb{P}$. As the graph of every new partial function in $F_{p}$ has empty intersection with $\left(n_{q} \times D_{q}\right)$, we conclude that $p \leq q$.

Proposition 2.3. In $V[G]$, we have that for any fixed $i \in \omega,\left(A_{\xi, i}\right)_{\xi<\omega_{1}}$ are pairwise disjoint nonempty sets and whenever $\left(i_{1}, \xi_{1}\right), \ldots,\left(i_{k}, \xi_{k}\right),(i, \xi)$ are distinct pairs, then

$$
A_{\xi, i} \backslash\left(A_{\xi_{1}, i_{1}} \cup \ldots \cup A_{\xi_{k}, i_{k}}\right) \neq \emptyset \text {. }
$$

Therefore, the Boolean algebra $\mathcal{B}$ generated by them is a c-algebra of cardinality $\omega_{1}$ and its Stone space $K$ is a uniform Eberlein compact space of weight $\omega_{1}$.

Proof. For all distinct $\left(i_{1}, \xi_{1}\right), \ldots,\left(i_{k}, \xi_{k}\right),(i, \xi)$, by Lemma 2.2 and the fact that $\left[n_{p} \times D_{p}\right]^{1} \subseteq F_{p}$ for any $p \in \mathbb{P}$, we have

$$
\{(i, \xi)\} \in A_{\xi, i} \backslash\left(A_{\xi_{1}, i_{1}} \cup \ldots \cup A_{\xi_{k}, i_{k}}\right) .
$$

So each $A_{\xi, i}$ is nonempty and the condition (c) of Definition 1.4 is satisfied. It also follows directly from the fact that the $f$ 's are functions and that any $\{(i, \xi)\} \in A_{\xi, i}$ that for a fixed $i \in \omega,\left(A_{\xi, i}\right)_{\xi<\omega_{1}}$ are pairwise disjoint and nonempty.

For each $(i, \xi) \in \omega \times \omega_{1}$, let $\dot{A}_{\xi, i}$ be a $\mathbb{P}$-name for $A_{\xi, i}$. 
Definition 2.4. Given $p_{1}=\left(n_{1}, D_{1}, F_{1}\right), p_{2}=\left(n_{2}, D_{2}, F_{2}\right) \in \mathbb{P}$, we say that they are isomorphic if $n_{1}=n_{2}$ and there is an order-preserving bijection $e: D_{1} \rightarrow D_{2}$ such that $\left.e\right|_{D_{1} \cap D_{2}}=i d$ and for all $f \in F n_{<\omega}\left(\omega, \omega_{1}\right), f \in F_{1}$ if and only if $e[f] \in F_{2}$, where $e[f](i)=e(f(i))$.

Lemma 2.5. Let $p_{k}=\left(n, D_{k}, F_{k}\right)$ in $\mathbb{P}$, for $1 \leq k \leq m$, be pairwise isomorphic conditions such that $\left(D_{k}\right)_{1 \leq k \leq m}$ is a $\Delta$-system with root $D$. Then, there is $p \in \mathbb{P}$, $p \leq p_{1}, \ldots, p_{m}$ such that for any distinct $1 \leq i, i^{\prime} \leq n$, any distinct $1 \leq k, k^{\prime} \leq m$ and any $\xi \in D_{k} \backslash D$ and $\xi^{\prime} \in D_{k^{\prime}} \backslash D$ we have

$$
p \Vdash \dot{A}_{\check{\xi}, i} \cap \dot{A}_{\check{\xi}^{\prime}, i^{\prime}}=\emptyset .
$$

Proof. We define $p=\left(n_{p}, D_{p}, F_{p}\right)$ by letting $n_{p}=n, D_{p}=D_{1} \cup \cdots \cup D_{m}$ and $F_{p}=F_{1} \cup \cdots \cup F_{m}$. Let us prove that $p \in \mathbb{P}$ and that $p \leq p_{k}$ for every $1 \leq k \leq m$.

As finite unions of finite sets, $D_{p}$ and $F_{p}$ are countable. Notice that $\left[n \times D_{p}\right]^{1}=$ $\left[n \times D_{1}\right]^{1} \cup \cdots \cup\left[n \times D_{m}\right]^{1} \subseteq F_{1} \cup \cdots \cup F_{m}=F_{p}$ and that $F_{p} \subseteq F n_{<\omega}\left(n, D_{1}\right) \cup$ $\cdots \cup F n_{<\omega}\left(n, D_{m}\right) \subseteq F n_{<\omega}\left(n, D_{p}\right)$, so that $p \in \mathbb{P}$.

Fix $1 \leq k \leq m$ and let us now show that $p \leq p_{k}$. By the definition of $p, n_{p}=n$, $D_{p} \supseteq D_{k}$ and $F_{p} \supseteq F_{k}$. Given $f \in F_{p},(\mathrm{P})$ of Definition 2.1 is trivially satisfied if $f \in F_{k}$. So, suppose $f \in F_{k^{\prime}} \backslash F_{k}$ for some $k^{\prime} \neq k$ and let $e$ be the order-preserving bijection from $D_{k^{\prime}}$ onto $D_{k}$. Since $p_{k}$ and $p_{k^{\prime}}$ are isomorphic and $f \in F_{k^{\prime}}$, we get that $g=e[f] \in F_{k}$. Let us prove that $G_{f} \cap\left(n_{k} \times D_{k}\right)=G_{g} \cap\left(n_{p} \times D\right) \subseteq G_{g}$ : given $(i, \xi) \in G_{f} \cap\left(n_{k} \times D_{k}\right)$, we have that $f(i)=\xi$ and since $f \in F_{k^{\prime}} \subseteq n_{p} \times D_{k^{\prime}}$, we get that $\xi \in D_{k} \cap D_{k^{\prime}}=D$. Then $g(i)=e[f](i)=e(f(i))=e(\xi)=\xi$, since $\xi \in D$. This proves that $G_{f} \cap\left(n_{k} \times D_{k}\right) \subseteq G_{g}$ and concludes the proof that $p \leq p_{k}$ for all $1 \leq k \leq m$.

Now let $1 \leq i, i^{\prime} \leq n$ be distinct, $1 \leq k<k^{\prime} \leq m$ and let $\xi \in D_{k} \backslash D$ and $\xi^{\prime} \in D_{k^{\prime}} \backslash D$. Note that in $F_{p}$ there is no function $f$ such that $f(i)=\xi$ and $f\left(i^{\prime}\right)=\xi^{\prime}$. Let $G$ be a $\mathbb{P}$-generic filter such that $p \in G$. Given any $p^{\prime} \in G$, there is $p^{\prime \prime} \in G$ such that $p^{\prime \prime} \leq p, p^{\prime}$. By $(\mathrm{P})$ of Definition 2.1. no function $g \in F_{p^{\prime \prime}}$ satisfies $g(i)=\xi$ and $g\left(i^{\prime}\right)=\xi^{\prime}$; hence there is no such function in $p^{\prime}$. It follows from the definition of the $A_{\xi, i}$ 's that $A_{\xi, i} \cap A_{\xi^{\prime}, i^{\prime}}=\emptyset$ in $V[G]$. Since $G$ was an arbitrary $\mathbb{P}$-generic filter containing $p$, we conclude that $p \Vdash \dot{A}_{\breve{\xi}, i} \cap \dot{A}_{\check{\xi}^{\prime}, i^{\prime}}=\emptyset$.

Theorem 2.6. $\mathbb{P}$ has precaliber $\omega_{1}$ and hence satisfies the c.c.c.

Proof. Let $\left(p_{\alpha}\right)_{\alpha<\omega_{1}} \subseteq \mathbb{P}$, where each $p_{\alpha}=\left(n_{\alpha}, D_{\alpha}, F_{\alpha}\right)$. By the $\Delta$-system lemma, we may assume that $\left(D_{\alpha}\right)_{\alpha<\omega_{1}}$ is a $\Delta$-system with root $D$. By standard counting arguments, we may assume without loss of generality that $n_{\alpha}=n$ for every $\alpha<\omega_{1}$ and some fixed $n \in \omega$. By thinning out using further counting arguments, we can assume that for every $\alpha<\beta<\omega_{1}, p_{\alpha}$ and $p_{\beta}$ are isomorphic. Now, given $\alpha_{1}<\ldots<\alpha_{n}<\omega_{1}$, by Lemma 2.5 there is $p \leq p_{\alpha_{1}}, \ldots, p_{\alpha_{n}}$.

Lemma 2.7. Let $p_{k}=\left(n, D_{k}, F_{k}\right)$ in $\mathbb{P}$, for $1 \leq k \leq m$, be pairwise isomorphic conditions such that $\left(D_{k}\right)_{1 \leq k \leq m}$ is a $\Delta$-system with root $D$. Given, for all $1 \leq k \leq$ $m, \xi_{k} \in D_{k} \backslash D$ and distinct $i_{k}<n$, there is $p \in \mathbb{P}, p \leq p_{1}, \ldots, p_{m}$ such that

$$
p \Vdash \dot{A}_{\check{\xi}_{1}, i_{1}} \cap \cdots \cap \dot{A}_{\check{\xi}_{m}, i_{m}} \neq \emptyset \text {. }
$$

Proof. We define $p=\left(n_{p}, D_{p}, F_{p}\right)$ by letting $n_{p}=n, D_{p}=D_{1} \cup \cdots \cup D_{m}$ and

$$
F_{p}=F_{1} \cup \cdots \cup F_{m} \cup\left\{f_{0}\right\},
$$


where $f_{0}=\left\{\left(i_{1}, \xi_{1}\right), \ldots,\left(i_{m}, \xi_{m}\right)\right\}$. Let us check that $p \in \mathbb{P}$ and that $p \leq p_{k}$ for every $1 \leq k \leq m$.

As finite unions of finite sets, $D_{p}$ and $F_{p}$ are finite. Notice that $\left[n \times D_{p}\right]^{1}=[n \times$ $\left.D_{1}\right]^{1} \cup \cdots \cup\left[n \times D_{m}\right]^{1} \subseteq F_{1} \cup \cdots \cup F_{m} \subseteq F_{p}$ and that $F_{p} \backslash\left\{f_{0}\right\} \subseteq F n_{<\omega}\left(n, D_{1}\right) \cup \cdots \cup$ $F n_{<\omega}\left(n, D_{m}\right) \subseteq F n_{<\omega}\left(n, D_{p}\right)$. Since $f_{0} \in F n_{<\omega}\left(n, D_{1} \cup \cdots \cup D_{m}\right) \subseteq F n_{<\omega}\left(n, D_{p}\right)$, we get that $p \in \mathbb{P}$.

Fix $1 \leq k \leq m$ and let us now verify that $p \leq p_{k}$. By the definition of $p, n_{p}=n$, $D_{p} \supseteq D_{k}$ and $F_{p} \supseteq F_{k}$. To check (P) of Definition 2.1] given $f \in F_{p}$, let us consider three cases:

Case 1. If $f \in F_{k}$, then $(\mathrm{P})$ is trivially satisfied.

Case 2. If $f \in F_{k^{\prime}} \backslash F_{k}$ for some $k^{\prime} \neq k$, let $e$ be the order-preserving bijection from $D_{k^{\prime}}$ onto $D_{k}$. Since $p_{k}$ and $p_{k^{\prime}}$ are isomorphic and $f \in F_{k^{\prime}}$, we get that $g=e[f] \in F_{k}$. Let us prove that $G_{f} \cap\left(n_{k} \times D_{k}\right)=G_{g} \cap\left(n_{p} \times D\right) \subseteq G_{g}$ : given $(i, \xi) \in G_{f} \cap\left(n_{k} \times D_{k}\right)$, we have that $f(i)=\xi$, and since $f \in F_{k^{\prime}} \subseteq n_{p} \times D_{k^{\prime}}$, we get that $\xi \in D_{k} \cap D_{k^{\prime}}=D$. Then $g(i)=e[f](i)=e(f(i))=e(\xi)=\xi$, since $\xi \in D$. This proves that $G_{f} \cap\left(n_{k} \times D_{k}\right) \subseteq G_{g}$, as we wanted.

Case 3. If $f=f_{0}=\left\{\left(i_{1}, \xi_{1}\right), \ldots,\left(i_{m}, \xi_{m}\right)\right\}$, then $G_{f} \cap\left(n_{k} \times D_{k}\right)=\left\{\left(i_{k}, \xi_{k}\right)\right\} \subseteq F_{k}$ by Definition 2.1.

Finally, since $f_{0} \in F_{p}, p$ forces that $\check{f}_{0} \in \dot{A}_{\check{\xi}_{1}, i_{1}} \cap \cdots \cap \dot{A}_{\check{\xi}_{m}, i_{m}}$, so that

$$
p \Vdash \dot{A}_{\check{\xi}_{1}, i_{1}} \cap \cdots \cap \dot{A}_{\check{\xi}_{m}, i_{m}} \neq \emptyset,
$$

which concludes the proof.

Definition 2.8. $\Sigma$ is the product of $\omega_{2}$ copies of $\mathbb{P}$, with finite supports, that is,

$$
\Sigma=\left\{\sigma: \operatorname{dom}(\sigma) \rightarrow \mathbb{P}: \operatorname{dom}(\sigma) \in\left[\omega_{2}\right]^{<\omega}\right\},
$$

ordered by $\sigma_{1} \leq \sigma_{2}$ if $\operatorname{dom}\left(\sigma_{1}\right) \supseteq \operatorname{dom}\left(\sigma_{2}\right)$ and for every $\alpha \in \operatorname{dom}\left(\sigma_{2}\right), \sigma_{1}(\alpha) \leq$ $\sigma_{2}(\alpha)$. Given $S \subseteq \omega_{2}$, let

$$
\Sigma_{S}=\{\sigma \in \Sigma: \operatorname{dom}(\sigma) \subseteq S\} .
$$

Proposition 2.9. Given $\gamma_{0} \in \omega_{2}$, the forcing $\Sigma_{\omega_{2} \backslash\left\{\gamma_{0}\right\}}$ is isomorphic to $\Sigma$.

Proof. Lift to $\mathbb{P}$ a bijection between $\omega_{2}$ and $\omega_{2} \backslash\left\{\gamma_{0}\right\}$.

Theorem 2.10. $V^{\Sigma} \vDash " \mathfrak{c}=\omega_{2}$ and there is no Banach space $X$ of density $\omega_{1}$ such that for every uniform Eberlein compact space $K$ of weight at most $\omega_{1}, C(K)$ can be isomorphically embedded into $X$ ".

Proof. We work in $V$. By contradiction, suppose that such a Banach space exists in $V^{\Sigma}$ and let $\dot{X}$ be a $\Sigma$-name for it. Since $\Sigma$ forces that $\dot{X}$ has density $\omega_{1}$, let $\left(\dot{v}_{\eta}\right)_{\eta<\omega_{1}}$ be a family of $\Sigma$-names such that $\Sigma \Vdash\left\{\dot{v}_{\eta}: \eta<\omega_{1}\right\}$ is a dense subset 1 of $\dot{X}$.

\footnotetext{
${ }^{1}$ Unfortunately, the Banach space $\dot{X}$ will not belong to any intermediate model: since new reals are being added all the time, no old complete metric space remains complete. So we cannot make use of any factorization of $\Sigma$ unless we are willing to deal with normed noncomplete spaces over the rationals and approximations (possibly nonlinear) of linear operators into its completion. We have chosen a simpler approach, in our opinion, and work in $V$ with the full product $\Sigma$.
} 
For each $\mathcal{F} \in\left[\omega_{1}\right]^{<\omega}$, let $\mathbb{A}_{\mathcal{F}} \subseteq \Sigma$ be a maximal antichain in $\Sigma$ such that for every $\sigma \in \mathbb{A}_{\mathcal{F}}$,

$$
\text { either } \sigma \Vdash\left\|\sum_{\eta \in \mathcal{F}} \dot{v}_{\eta}\right\|>2 \text { or } \sigma \Vdash\left\|\sum_{\eta \in \mathcal{F}} \dot{v}_{\eta}\right\| \leq 2 \text {. }
$$

Since $\Sigma$ is c.c.c., each $\mathbb{A}_{\mathcal{F}}$ is countable and for each $\sigma \in \mathbb{A}_{\mathcal{F}},|\operatorname{dom}(\sigma)|<\omega$. Then, the set

$$
\bigcup\left\{\operatorname{dom}(\sigma): \sigma \in \mathbb{A}_{\mathcal{F}} \text { for some } \mathcal{F} \in\left[\omega_{1}\right]^{<\omega}\right\}
$$

has cardinality at most $\omega_{1}$. Fix $\gamma_{0} \in \omega_{2}$ such that for every $\mathcal{F} \in\left[\omega_{1}\right]^{<\omega}$ and every $\sigma \in \mathbb{A}_{\mathcal{F}}, \gamma_{0} \notin \operatorname{dom}(\sigma)$.

Since $\Sigma \sim \Sigma_{\omega_{2} \backslash\left\{\gamma_{0}\right\}} \times \Sigma_{\left\{\gamma_{0}\right\}} \sim \Sigma_{\omega_{2} \backslash\left\{\gamma_{0}\right\}} \times \mathbb{P}$, let us consider in $V^{\Sigma_{\omega_{2} \backslash\left\{\gamma_{0}\right\}} \times \Sigma_{\left\{\gamma_{0}\right\}}}$ the Stone space $K_{\gamma_{0}}$ of the algebra generated by the family $\left(A_{\xi, i}\left(\gamma_{0}\right)\right)_{\xi \in \omega_{1}, i \in \omega}$ added by $\Sigma_{\left\{\gamma_{0}\right\}} \sim \mathbb{P}$ over $V^{\Sigma_{\omega_{2} \backslash\left\{\gamma_{0}\right\}}}$. By Proposition 2.3. $K_{\gamma_{0}}$ is a uniform Eberlein compact space of weight $\omega_{1}$. Let us show that $C\left(K_{\gamma_{0}}\right)$ does not embed isomorphically into $\dot{X}$. For each $(i, \xi) \in \omega \times \omega_{1}$, let $\dot{A}_{\xi, i}$ be a $\Sigma$-name for $A_{\xi, i}\left(\gamma_{0}\right)$.

Suppose there is $\dot{T}: C\left(K_{\gamma_{0}}\right) \rightarrow \dot{X}$ an isomorphic embedding and, without loss of generality, assume that $\Sigma \Vdash\|\dot{T}\|=1$. Let $p_{0} \in \Sigma$. Now find $p_{1} \leq p_{0}$ and $m \in \omega$ such that $p_{1} \Vdash 3 \cdot\left\|\dot{T}^{-1}\right\|<\check{m}$.

For each $\alpha<\omega_{1}$, let $\left\{\xi_{k}(\alpha): 1 \leq k \leq m\right\} \subseteq \omega_{1} \backslash \alpha$ have cardinality $m$ and take $\sigma_{\alpha} \in \Sigma$ with $\sigma_{\alpha} \leq p_{1}$ and $\eta_{1}(\alpha), \ldots, \eta_{m}(\alpha) \in \omega_{1}$ such that

$$
\sigma_{\alpha} \Vdash \forall 1 \leq k \leq m \quad\left\|\dot{T}\left(\chi_{\dot{A}_{\check{\xi}_{k}(\alpha), k}\left(\gamma_{0}\right)}\right)-\dot{v}_{\check{\eta}_{k}(\alpha)}\right\|<\frac{1}{m} .
$$

Let $s_{\alpha}=\left.\sigma_{\alpha}\right|_{\omega_{2} \backslash\left\{\gamma_{0}\right\}} \in \Sigma_{\omega_{2} \backslash\left\{\gamma_{0}\right\}}$ and $\sigma_{\alpha}\left(\gamma_{0}\right)=\left(n_{\alpha}, D_{\alpha}, F_{\alpha}\right)$. Without loss of generality, we may assume that $\xi_{1}(\alpha), \ldots, \xi_{m}(\alpha) \in D_{\alpha}$. Using the $\Delta$-system lemma, we may assume that $\left(D_{\alpha}\right)_{\alpha<\omega_{1}}$ is a $\Delta$-system with root $D$ and that $\xi_{1}(\alpha), \ldots, \xi_{m}(\alpha) \in$ $D_{\alpha} \backslash D$ for each $\alpha<\omega_{1}$ and for all $\alpha<\omega_{1}$ the conditions $\sigma_{\alpha}\left(\gamma_{0}\right)$ are pairwise isomorphic.

By Proposition [2.9, $\Sigma_{\omega_{2} \backslash\left\{\gamma_{0}\right\}}$ is isomorphic to $\Sigma$ and since, by the productivity of precaliber $\omega_{1}$ and Lemma 2.6, $\Sigma$ has precaliber $\omega_{1}$, so does $\Sigma_{\omega_{2} \backslash\left\{\gamma_{0}\right\}}$. So, given $\alpha_{1}<\cdots<\alpha_{m}$, there is $s \in \Sigma_{\omega_{2} \backslash\left\{\gamma_{0}\right\}}$ such that $s \leq s_{\alpha_{1}}, \ldots, s_{\alpha_{m}}$.

Let $\mathcal{F}=\left\{\eta_{1}\left(\alpha_{1}\right), \ldots, \eta_{m}\left(\alpha_{m}\right)\right\}$ and since $\mathbb{A}_{\mathcal{F}}$ is a maximal antichain in $\Sigma$ which is contained in $\Sigma_{\omega_{2} \backslash\left\{\gamma_{0}\right\}}, \mathbb{A}_{\mathcal{F}}$ is also a maximal antichain in $\Sigma_{\omega_{2} \backslash\left\{\gamma_{0}\right\}}$. Then, there is $s^{\prime} \in \mathbb{A}_{\mathcal{F}}$ and $s^{\prime \prime} \in \Sigma_{\omega_{2} \backslash\left\{\gamma_{0}\right\}}$ such that $s^{\prime \prime} \leq s, s^{\prime}$.

Since $s^{\prime \prime} \leq s^{\prime}$ and $s^{\prime} \in \mathbb{A}_{\mathcal{F}}$, either

$$
s^{\prime \prime} \Vdash\left\|\dot{v}_{\check{\eta}_{1}\left(\alpha_{1}\right)}+\cdots+\dot{v}_{\check{\eta}_{m}\left(\alpha_{m}\right)}\right\| \leq 2
$$

or

$$
s^{\prime \prime} \Vdash\left\|\dot{v}_{\check{\eta}_{1}\left(\alpha_{1}\right)}+\cdots+\dot{v}_{\check{\eta}_{m}\left(\alpha_{m}\right)}\right\|>2 .
$$

Let us now get a contradiction.

Case 1. $s^{\prime \prime}$ forces that $\left\|\dot{v}_{\check{\eta}_{1}\left(\alpha_{1}\right)}+\cdots+\dot{v}_{\check{\eta}_{m}\left(\alpha_{m}\right)}\right\|>2$.

In this case, let $p \in \mathbb{P}$ be such that $p \leq \sigma_{\alpha_{1}}\left(\gamma_{0}\right), \ldots, \sigma_{\alpha_{m}}\left(\gamma_{0}\right)$ obtained by Lemma 2.5 and let $\sigma=\left(s^{\prime \prime}, p\right) \in \Sigma$. Then, $\sigma \leq s^{\prime \prime}, p$ and $\sigma \leq \sigma_{\alpha_{1}}, \ldots, \sigma_{\alpha_{m}}$. So,

so that

$$
\sigma \Vdash \forall 1 \leq k<k^{\prime} \leq m \quad \dot{A}_{\check{\xi}_{k}\left(\alpha_{k}\right), i_{k}}\left(\gamma_{0}\right) \cap \dot{A}_{\check{\xi}_{k^{\prime}}\left(\alpha_{k^{\prime}}\right), i_{k^{\prime}}}\left(\gamma_{0}\right)=\emptyset
$$

$$
\sigma \Vdash\left\|\chi_{\left[\dot{A}_{\check{\xi}_{1}\left(\alpha_{1}\right), i_{1}}\left(\gamma_{0}\right)\right]}+\cdots+\chi_{\left[\dot{A}_{\check{\xi}_{m}\left(\alpha_{m}\right), i_{m}}\left(\gamma_{0}\right)\right]}\right\|=1 .
$$


Since $\sigma \leq \sigma_{\alpha_{1}}, \ldots, \sigma_{\alpha_{m}}$

$$
\sigma \Vdash \forall 1 \leq k \leq m \quad\left\|\dot{T}\left(\chi_{\left[\dot{A}_{\check{\xi}_{k}\left(\alpha_{k}\right), i_{k}}\left(\gamma_{0}\right)\right]}\right)-\dot{v}_{\check{\eta}_{k}\left(\alpha_{k}\right)}\right\|<\frac{1}{m},
$$

so that, using the fact that $\Sigma \Vdash\|\dot{T}\|=1$, we get that

$$
\begin{aligned}
\sigma \Vdash & \left\|\dot{v}_{\check{\eta}_{1}\left(\alpha_{1}\right)}+\cdots+\dot{v}_{\check{\eta}_{m}\left(\alpha_{m}\right)}\right\| \\
& \leq\left\|\sum_{k=1}^{m}\left(\dot{v}_{\check{\eta}_{k}\left(\alpha_{k}\right)}-\dot{T}\left(\chi_{\left[\dot{A}_{\check{\xi}_{k}\left(\alpha_{k}\right), i_{k}}\left(\gamma_{0}\right)\right]}\right)\right)+\sum_{k=1}^{m} \dot{T}\left(\chi_{\left[\dot{A}_{\check{\xi}_{k}\left(\alpha_{k}\right), i_{k}}\left(\gamma_{0}\right)\right]}\right)\right\| \\
& \leq\left\|\chi_{\left[\dot{A}_{\check{\xi}_{1}\left(\alpha_{1}\right), i_{1}}\left(\gamma_{0}\right)\right]}+\cdots+\chi_{\left[\dot{A}_{\check{\xi}_{m}\left(\alpha_{m}\right), i_{m}}\left(\gamma_{0}\right)\right]}\right\|+m \cdot \frac{1}{m} \leq 2,
\end{aligned}
$$

contradicting the fact that $\sigma \leq s^{\prime \prime}$ and

$$
s^{\prime \prime} \Vdash\left\|\dot{v}_{\check{\eta}_{1}\left(\alpha_{1}\right)}+\cdots+\dot{v}_{\check{\eta}_{m}\left(\alpha_{m}\right)}\right\|>2 .
$$

Case 2. $s^{\prime \prime}$ forces that $\left\|\dot{v}_{\check{\eta}_{1}\left(\alpha_{1}\right)}+\cdots+\dot{v}_{\check{\eta}_{m}\left(\alpha_{m}\right)}\right\| \leq 2$.

In this case, let $p \in \mathbb{P}$ be such that $p \leq \sigma_{\alpha_{1}}\left(\gamma_{0}\right), \ldots, \sigma_{\alpha_{m}}\left(\gamma_{0}\right)$ obtained by Lemma 2.7 and let $\sigma=\left(s^{\prime \prime}, p\right) \in \Sigma$. Then, $\sigma \leq s^{\prime \prime}, p$ and $\sigma \leq \sigma_{\alpha_{1}}, \ldots, \sigma_{\alpha_{m}}$. So,

so that

$$
\sigma \Vdash \dot{A}_{\check{\xi}_{1}\left(\alpha_{1}\right), i_{1}}\left(\gamma_{0}\right) \cap \cdots \cap \dot{A}_{\check{\xi}_{m}\left(\alpha_{m}\right), i_{m}}\left(\gamma_{0}\right) \neq \emptyset
$$

Since $\sigma \leq \sigma_{\alpha_{1}}, \ldots, \sigma_{\alpha_{m}}$

$$
\sigma \Vdash\left\|\chi_{\left[\dot{A}_{\check{\xi}_{1}\left(\alpha_{1}\right), i_{1}}\left(\gamma_{0}\right)\right]}+\cdots+\chi_{\left[\dot{A}_{\check{\xi}_{m}\left(\alpha_{m}\right), i_{m}}\left(\gamma_{0}\right)\right]}\right\|=m .
$$

$$
\sigma \Vdash \forall 1 \leq k \leq m \quad\left\|\dot{T}\left(\chi_{\left[\dot{A}_{\check{\xi}_{k}\left(\alpha_{k}\right), i_{k}}\left(\gamma_{0}\right)\right]}\right)-\dot{v}_{\check{\eta}_{k}\left(\alpha_{k}\right)}\right\|<\frac{1}{m},
$$

so that, using the fact that $\sigma \leq s^{\prime \prime}$, we get that

$$
\begin{aligned}
\sigma \Vdash & \left\|\dot{T}\left(\chi_{\left[\dot{A}_{\check{\xi}_{1}\left(\alpha_{1}\right), i_{1}}\right]}+\cdots+\chi_{\left[\dot{A}_{\left.\check{\xi}_{m}\left(\alpha_{m}\right), i_{m}\right]}\right]}\right)\right\| \\
& \leq\left\|\sum_{k=1}^{m}\left(\dot{T}\left(\chi_{\left[\dot{A}_{\check{\xi}_{k}\left(\alpha_{k}\right), i_{k}}\left(\gamma_{0}\right)\right]}\right)-\dot{v}_{\check{\eta}_{k}\left(\alpha_{k}\right)}\right)+\sum_{k=1}^{m} \dot{v}_{\check{\eta}_{k}\left(\alpha_{k}\right)}\right\| \\
& \leq\left\|\dot{v}_{\check{\eta}_{1}\left(\alpha_{1}\right)}+\cdots+\dot{v}_{\check{\eta}_{m}\left(\alpha_{m}\right)}\right\|+m \cdot \frac{1}{m} \leq 3,
\end{aligned}
$$

which contradicts the fact that $\Sigma \Vdash 3 \cdot\left\|\dot{T}^{-1}\right\|<m$.

Since the condition $p_{0} \in \Sigma$ was arbitrary, we showed that a dense subset of $\Sigma$ forces the nonexistence of the embedding $\dot{T}$; hence it does not exist in $V[G]$.

\section{Density $\mathfrak{c}$}

This section is devoted to the proof of the main result (Theorem 1.3) for the case $\kappa=\mathfrak{c}$. The following partial order will approximate the generic c-algebras of cardinality $\omega_{2}=\mathfrak{c}$, one of which will induce a Banach space UG without an isomorphic embedding into a given Banach space.

Definition 3.1. $\mathbb{Q}$ is the forcing notion of conditions $q=\left(D_{q}, F_{q}\right)$, where $D_{q} \in$ $\left[\omega_{2}\right] \leq \omega$ and $F_{q} \subseteq F n_{\leq \omega}\left(\omega, D_{q}\right)$ are such that $\left|F_{q}\right| \leq \omega$ and $\left[\omega \times D_{q}\right]^{1} \subseteq F_{q}$, ordered by $p \leq q$ if $D_{p} \supseteq D_{q}, F_{p} \supseteq F_{q}$ and

(Q) Given $f \in F_{p}$, there is $g \in F_{q}$ such that $G_{f} \cap\left(\omega \times D_{q}\right) \subseteq G_{g}$.

Proposition 3.2. $\mathbb{Q}$ is $\sigma$-closed. 
Proof. Let $\left(q_{n}\right)_{n \in \omega} \subseteq \mathbb{Q}$ be such that $q_{n+1} \leq q_{n}$ for all $n \in \omega$, where each $q_{n}=$ $\left(D_{n}, F_{n}\right)$. Define $q=\left(D_{q}, F_{q}\right)$ by $D_{q}=\bigcup_{n \in \omega} D_{n}$ and $F_{q}=\bigcup_{n \in \omega} F_{n}$. As countable unions of countable sets, $D_{q}$ and $F_{q}$ are countable. Also, $\left[\omega \times D_{q}\right]^{1}=\bigcup_{n \in \omega}([\omega \times$ $\left.\left.D_{n}\right]^{1}\right) \subseteq \bigcup_{n \in \omega} F_{n}=F_{q}$ and $F_{q} \subseteq \bigcup_{n \in \omega} F n_{\leq \omega}\left(\omega, D_{n}\right) \subseteq F n_{\leq \omega}\left(\omega, D_{q}\right)$, so that $q \in \mathbb{Q}$.

Fix $n \in \omega$ and let us now prove that $q \leq q_{n}$. Clearly $D_{q} \supseteq D_{n}$ and $F_{q} \supseteq D_{n}$. To prove (Q) of Definition 3.1, given $f \in F_{q}$, there is $k \in \omega$ such that $f \in F_{k}$. If $k \leq n$, then $q_{n} \leq q_{k}$, so that $f \in F_{k} \subseteq F_{n}$ and we are done. If $k>n$, since $q_{k} \leq q_{n}$, there is $g \in F_{n}$ such that $G_{f} \cap\left(\omega \times D_{n}\right) \subseteq G_{g}$. If suffices now to notice that $g \in F_{n} \subseteq F_{q}$.

Lemma 3.3. For every $\xi \in \omega_{2}$ and every $q \in \mathbb{Q}$ there is $p \leq q$ such that $\xi \in D_{p}$.

Proof. Let $D_{q}=D_{p} \cup\{\xi\}, F_{p}=F_{q} \cup[\omega \times\{\xi\}]^{1}$. Clearly $p \in \mathbb{P}$. As the graphs of all new partial functions in $F_{p}$ have empty intersections with $\left(\omega \times D_{q}\right)$ we conclude that $p \leq q$.

Given a model $V$ and a $\mathbb{Q}$-generic filter $G$ over $V$, for each $\xi \in \omega_{2}$ and each $i \in \omega$, we define in $V[G]$ the following set:

$$
A_{\xi, i}=\left\{f \in F n_{\leq \omega}\left(\omega, \omega_{2}\right): \exists q \in G \text { such that } f \in F_{q} \text { and } f(i)=\xi\right\} .
$$

Let $\mathcal{B}$ be the Boolean algebra generated by $\left\{A_{\xi, i}:(i, \xi) \in \omega \times \omega_{2}\right\}$.

Proposition 3.4. In $V[G]$, we have that for a fixed $i \in \omega,\left(A_{\xi, i}\right)_{\xi<\omega_{2}}$ are pairwise disjoint nonempty sets and whenever $\left(i_{1}, \xi_{1}\right), \ldots,\left(i_{k}, \xi_{k}\right),(i, \xi)$ are distinct pairs, then

$$
A_{\xi, i} \backslash\left(A_{\xi_{1}, i_{1}} \cup \ldots \cup A_{\xi_{k}, i_{k}}\right) \neq \emptyset \text {. }
$$

Therefore, the Boolean algebra $\mathcal{B}$ generated by them is a c-algebra of cardinality $\omega_{2}$ and its Stone space $K$ is a uniform Eberlein compact space of weight $\omega_{2}$.

Proof. The proof is similar to the proof of Proposition 2.3 .

For each $(i, \xi) \in \omega \times \omega_{2}$, let $\dot{A}_{\xi, i}$ be a $\mathbb{Q}$-name for $A_{\xi, i}$.

Definition 3.5. Given $q_{1}=\left(D_{1}, F_{1}\right), q_{2}=\left(D_{2}, F_{2}\right) \in \mathbb{Q}$, we say that they are isomorphic if there is an order-preserving bijection $e: D_{1} \rightarrow D_{2}$ such that $\left.e\right|_{D_{1} \cap D_{2}}=$ $i d$ and for all $f \in F n_{\leq \omega}\left(\omega, \omega_{2}\right), f \in F_{1}$ if and only if $e[f] \in F_{2}$, where $e[f](i)=$ $e(f(i))$.

Lemma 3.6. Let $q_{k}=\left(D_{k}, F_{k}\right)$ in $\mathbb{Q}$, for $1 \leq k \leq m$, be pairwise isomorphic conditions such that $\left(D_{k}\right)_{1 \leq k \leq m}$ is a $\Delta$-system with a countable root $D$. Then, there is $q \in \mathbb{Q}, q \leq q_{1}, \ldots, q_{m}$ such that for any distinct $i, i^{\prime} \in \omega$, any distinct $1 \leq k, k^{\prime} \leq m$ and any $\xi \in D_{k} \backslash D$ and $\xi^{\prime} \in D_{k^{\prime}} \backslash D$ we have

$$
q \Vdash \dot{A}_{\check{\xi}, i} \cap \dot{A}_{\check{\xi}^{\prime}, i^{\prime}}=\emptyset .
$$

Proof. We define $q=\left(D_{q}, F_{q}\right)$ by letting $D_{q}=D_{1} \cup \cdots \cup D_{m}$ and $F_{q}=F_{1} \cup \cdots \cup F_{m}$. Let us prove that $q \in \mathbb{Q}$ and that $q \leq q_{k}$ for every $1 \leq k \leq m$.

As finite unions of countable sets, $D_{q}$ and $F_{q}$ are countable. Notice that $\omega \times D_{q}=$ $\left(\omega \times D_{1}\right) \cup \cdots \cup\left(\omega \times D_{m}\right) \subseteq F_{1} \cup \cdots \cup F_{m}=F_{q}$ and that $F_{q} \subseteq F n_{\leq \omega}\left(\omega, D_{1}\right) \cup \cdots \cup$ $F n_{\leq \omega}\left(\omega, D_{m}\right) \subseteq F n_{\leq \omega}\left(\omega, D_{q}\right)$, so that $q \in \mathbb{Q}$.

Fix $1 \leq k \leq m$ and let us now show that $q \leq q_{k}$. By the definition of $q, D_{q} \supseteq D_{k}$ and $F_{q} \supseteq F_{k}$. Given $f \in F_{q}$, (Q) of Definition 3.1 is trivially satisfied if $f \in F_{k}$. So, suppose $f \in F_{k^{\prime}} \backslash F_{k}$ for some $k^{\prime} \neq k$ and let $e$ be the order-preserving bijection 
from $D_{k^{\prime}}$ onto $D_{k}$. Since $q_{k}$ and $q_{k^{\prime}}$ are isomorphic and $f \in F_{k^{\prime}}$, we get that $g=e[f] \in F_{k}$. Let us prove that $G_{f} \cap\left(\omega \times D_{k}\right)=G_{g} \cap(\omega \times D) \subseteq G_{g}$ : given $(i, \xi) \in G_{f} \cap\left(\omega \times D_{k}\right)$, we have that $f(i)=\xi$ and since $f \in F_{k^{\prime}} \subseteq \omega \times D_{k^{\prime}}$, we get that $\xi \in D_{k} \cap D_{k^{\prime}}=D$. Then $g(i)=e[f](i)=e(f(i))=e(\xi)=\xi$, since $\xi \in D$. This proves that $G_{f} \cap\left(\omega \times D_{k}\right) \subseteq G_{g}$ and concludes the proof.

Now let $i, i^{\prime} \in \omega$ be distinct, $1 \leq k<k^{\prime} \leq m$ and let $\xi \in D_{k} \backslash D$ and $\xi^{\prime} \in D_{k^{\prime}} \backslash D$. Note that in $F_{q}$ there is no function $f$ such that $f(i)=\xi$ and $f\left(i^{\prime}\right)=\xi^{\prime}$. Let $G$ be a $\mathbb{Q}$-generic filter such that $q \in G$. Given any $q^{\prime} \in G$, there is $q^{\prime \prime} \in G$ such that $q^{\prime \prime} \leq q, q^{\prime}$. By Definition 3.1 (Q), no function $g \in F_{q^{\prime \prime}}$ satisfies $g(i)=\xi$ and $g\left(i^{\prime}\right)=\xi^{\prime}$; hence there is no such function in $F_{q^{\prime}}$. It follows from the definition of the $A_{\xi, i}$ 's that $A_{\xi, i} \cap A_{\xi^{\prime}, i^{\prime}}=\emptyset$ in $V[G]$. Since $G$ was an arbitrary $\mathbb{Q}$-generic filter containing $q$, we conclude that $q \Vdash \dot{A}_{\check{\xi}_{, j}} \cap \dot{A}_{\check{\xi}^{\prime}, i^{\prime}}=\emptyset$.

Theorem 3.7. Assuming CH, given $\left(q_{\alpha}\right)_{\alpha<\omega_{2}} \subseteq \mathbb{Q}$, there is $I \in\left[\omega_{2}\right]^{\omega_{2}}$ such that for every $\alpha_{1}<\cdots<\alpha_{m} \in I$, there is $q \in \mathbb{Q}, q \leq q_{\alpha_{1}}, \ldots, q_{\alpha_{m}}$. In particular, $\mathbb{Q}$ satisfies the $\omega_{2}$-c.c.

Proof. Given $\left(q_{\alpha}\right)_{\alpha<\omega_{2}} \subseteq \mathbb{Q}$, using $\mathrm{CH}$, the $\Delta$-system lemma for countable sets (see [11]) and standard counting arguments we can find a set $I \in\left[\omega_{2}\right]^{\omega_{2}}$ such that $\left(q_{\alpha}\right)_{\alpha \in I}$ are pairwise isomorphic. Now, given $\alpha_{1}<\cdots<\alpha_{m} \in A$, by Lemma 2.5 there is $q \in \mathbb{Q}, q \leq q_{\alpha_{1}}, \ldots, q_{\alpha_{m}}$, which concludes the proof.

Lemma 3.8. Let $q_{k}=\left(D_{k}, F_{k}\right)$ in $\mathbb{Q}$, for $1 \leq k \leq m$, be pairwise isomorphic conditions such that $\left(D_{k}\right)_{1 \leq k \leq m}$ is a $\Delta$-system with a countable root D. Given, for all $1 \leq k \leq m, \xi_{k} \in D_{k} \backslash D$ and distinct $i_{k} \in \omega$, there is $q \in \mathbb{Q}, q \leq q_{1}, \ldots, q_{m}$ such that

$$
q \Vdash \dot{A}_{\check{\xi}_{1}, i_{1}} \cap \cdots \cap \dot{A}_{\check{\xi}_{m}, i_{m}} \neq \emptyset .
$$

Proof. We define $q=\left(D_{q}, F_{q}\right)$ by letting $D_{q}=D_{1} \cup \cdots \cup D_{m}$ and

$$
F_{q}=F_{1} \cup \cdots \cup F_{m} \cup\left\{f_{0}\right\},
$$

where $f_{0}=\left\{\left(i_{1}, \xi_{1}\right), \ldots,\left(i_{m}, \xi_{m}\right)\right\}$. Let us check that $q \in \mathbb{Q}$ and that $q \leq q_{k}$ for every $1 \leq k \leq m$.

As finite unions of countable sets, $D_{q}$ and $F_{q}$ are countable. Notice that $[\omega \times$ $\left.D_{q}\right]^{1}=\left[\omega \times D_{1}\right]^{1} \cup \cdots \cup\left[\omega \times D_{m}\right]^{1} \subseteq F_{1} \cup \cdots \cup F_{m} \subseteq F_{q}$ and that $F_{q} \backslash\left\{f_{0}\right\} \subseteq$ $F n_{\leq \omega}\left(\omega, D_{1}\right) \cup \cdots \cup F n_{\leq \omega}\left(\omega, D_{m}\right) \subseteq F n_{\leq \omega}\left(\omega, D_{q}\right)$. Since $f_{0} \in F n_{\leq \omega}\left(\omega, D_{1} \cup \cdots \cup\right.$ $\left.D_{m}\right) \subseteq F n_{\leq \omega}\left(\omega, D_{q}\right)$, we get that $q \in \mathbb{Q}$.

Fix $1 \leq k \leq m$ and let us now verify that $q \leq q_{k}$. By the definition of $q, D_{q} \supseteq D_{k}$ and $F_{q} \supseteq F_{k}$. To check (Q) of Definition 3.1] given $f \in F_{q}$, let us consider three cases:

Case 1 . If $f \in F_{k}$, then (Q) is trivially satisfied.

Case 2. If $f \in F_{k^{\prime}} \backslash F_{k}$ for some $k^{\prime} \neq k$, let $e$ be the order-preserving bijection from $D_{k^{\prime}}$ onto $D_{k}$. Since $q_{k}$ and $q_{k^{\prime}}$ are isomorphic and $f \in F_{k^{\prime}}$, we get that $g=e[f] \in F_{k}$. Let us prove that $G_{f} \cap\left(\omega \times D_{k}\right)=G_{g} \cap(\omega \times D) \subseteq G_{g}$ : given $(i, \xi) \in G_{f} \cap\left(\omega \times D_{k}\right)$, we have that $f(i)=\xi$ and since $f \in F_{k^{\prime}} \subseteq \omega \times D_{k^{\prime}}$, we get that $\xi \in D_{k} \cap D_{k^{\prime}}=D$. Then $g(i)=e[f](i)=e(f(i))=e(\xi)=\xi$, since $\xi \in D$. This proves that $G_{f} \cap\left(\omega \times D_{k}\right) \subseteq G_{g}$, as we wanted.

Case 3. If $f=f_{0}=\left\{\left(i_{1}, \xi_{1}\right), \ldots,\left(i_{m}, \xi_{m}\right)\right\}$, then $G_{f} \cap\left(\omega \times D_{k}\right)=\left\{\left(i_{k}, \xi_{k}\right)\right\} \subseteq F_{k}$ by Definition 3.1 . 
Finally, since $f_{0} \in F_{q}, q$ forces that $\check{f}_{0} \in \dot{A}_{\check{\xi}_{1}, i_{1}} \cap \cdots \cap \dot{A}_{\check{\xi}_{m}, i_{m}}$ and we get that

$$
q \Vdash \dot{A}_{\check{\xi}_{1}, i_{1}} \cap \cdots \cap \dot{A}_{\check{\xi}_{m}, i_{m}} \neq \emptyset,
$$

which concludes the proof.

Definition 3.9. $\Pi$ is the product of $\omega_{3}$ copies of $\mathbb{Q}$, with countable supports; that is:

$$
\Pi=\left\{\pi: \operatorname{dom}(\pi) \rightarrow \mathbb{Q}: \operatorname{dom}(\pi) \in\left[\omega_{3}\right]^{\leq \omega}\right\},
$$

ordered by $\pi_{1} \leq \pi_{2}$ if $\operatorname{dom}\left(\pi_{1}\right) \supseteq \operatorname{dom}\left(\pi_{2}\right)$ and for every $\alpha \in \operatorname{dom}\left(\pi_{2}\right), \pi_{1}(\alpha) \leq \mathbb{Q}$ $\pi_{2}(\alpha)$. Given $S \subseteq \omega_{3}$, let

$$
\Pi_{S}=\{\pi \in \Pi: \operatorname{dom}(\pi) \subseteq S\} .
$$

$\mathbb{R}$ is the product of Cohen forcing adding $\omega_{2}$ Cohen reals with $\Pi$, that is,

$$
\mathbb{R}=F n_{<\omega}\left(\omega_{2}, 2\right) \times \Pi \text {. }
$$

Proposition 3.10. Given $\gamma_{0} \in \omega_{3}$, the forcing $\Pi_{\omega_{3} \backslash\left\{\gamma_{0}\right\}}$ is isomorphic to $\Pi$ and, therefore,

$$
\mathbb{R}=F n_{<\omega}\left(\omega_{2}, 2\right) \times \Pi \sim F n_{<\omega}\left(\omega_{2}, 2\right) \times \Pi_{\omega_{3} \backslash\left\{\gamma_{0}\right\}} \times \mathbb{Q} \sim \mathbb{R} \times \mathbb{Q} .
$$

Proof. Lift a bijection between $\omega_{3}$ and $\omega_{3} \backslash\left\{\gamma_{0}\right\}$ to an isomorphism of $\Pi$ and $\Pi_{\omega_{3} \backslash\left\{\gamma_{0}\right\}} \cdot$

Given a model $V$ of $\mathrm{CH}$ and an $\mathbb{R}$-generic filter $G$ over $V$, for each $\gamma_{0} \in \omega_{3}$, each $\xi \in \omega_{2}$ and each $i \in \omega$, we define in $V[G]$ the following set:

$A_{\xi, i}\left(\gamma_{0}\right)=\left\{f \in F n_{\leq \omega}\left(\omega, \omega_{2}\right) \cap V: \exists(C, \pi) \in G\right.$ such that $f \in F_{\pi\left(\gamma_{0}\right)}$ and $\left.f(i)=\xi\right\}$.

Let $\mathcal{B}_{\gamma_{0}}$ be a subalgebra of the Boolean algebra $\wp\left(F n_{\leq \omega}\left(\omega, \omega_{2}\right)\right)$ generated by $\left\{A_{\xi, i}\left(\gamma_{0}\right):(i, \xi) \in \omega \times \omega_{2}\right\}$.

Proposition 3.11. In $V[G]$, we have that for every $\gamma_{0} \in \omega_{3}$ and a fixed $i \in$ $\omega,\left(A_{\xi, i}\left(\gamma_{0}\right)\right)_{\xi<\omega_{2}}$ are pairwise disjoint nonempty sets and whenever $\left(i_{1}, \xi_{1}\right), \ldots$, $\left(i_{k}, \xi_{k}\right),(i, \xi)$ are distinct pairs, then

$$
A_{\xi, i}\left(\gamma_{0}\right) \backslash\left(A_{\xi_{1}, i_{1}}\left(\gamma_{0}\right) \cup \ldots \cup A_{\xi_{k}, i_{k}}\left(\gamma_{0}\right)\right) \neq \emptyset .
$$

Therefore, the Boolean algebra $\mathcal{B}_{\gamma_{0}}$ generated by them is a c-algebra of cardinality $\omega_{2}$ and its Stone space $K_{\gamma_{0}}$ is a uniform Eberlein compact space of weight $\omega_{2}$.

Proof. Fix $\gamma_{0} \in \omega_{3}$. The proof is similar to the proof of Proposition 2.3, using Lemma 3.3

Since now we work in $V$, for each $\gamma_{0} \in \omega_{3}$ and each $(i, \xi) \in \omega \times \omega_{2}$, let $\dot{A}_{\xi, i}\left(\gamma_{0}\right)$ be an $\mathbb{R}$-name for $A_{\xi, i}\left(\gamma_{0}\right)$.

Lemma 3.12. Assuming $C H$, given $\left(r_{\alpha}\right)_{\alpha<\omega_{2}} \subseteq \mathbb{R}$, there is $I \in\left[\omega_{2}\right]^{\omega_{2}}$ such that for every $\alpha_{1}<\cdots<\alpha_{m} \in I$, there is $r \in \mathbb{R}, r \leq r_{\alpha_{1}}, \ldots, r_{\alpha_{m}}$. In particular, $\mathbb{R}$ is $\omega_{2}$-c.c.

Proof. Let $\left(r_{\alpha}\right)_{\alpha<\omega_{2}} \subseteq \mathbb{R}$, where each $r_{\alpha}=\left(C_{\alpha}, \pi_{\alpha}\right)$. Let $B_{\alpha}=\operatorname{dom}\left(\pi_{\alpha}\right) \in\left[\omega_{3}\right]^{\leq \omega}$. Using $\mathrm{CH}$, the $\Delta$-system lemma for countable sets and standard counting arguments we can find a set $I \in\left[\omega_{2}\right]^{\omega_{2}}$ such that

- $\left(B_{\alpha}\right)_{\alpha \in I}$ is a $\Delta$-system with countable root $B$,

- $\left(\operatorname{dom}\left(C_{\alpha}\right)\right)_{\alpha \in I}$ is a $\Delta$-system with finite root $\Delta$,

- $\left.C_{\alpha}\right|_{\Delta}=\left.C_{\beta}\right|_{\Delta}$ for any $\alpha<\beta \in I$, 
- for every $\left.\xi \in B,\left(D_{r_{\alpha}(\xi)}\right)\right)_{\alpha \in I}$ is a $\Delta$-system with countable root $D_{\xi}$

- and $\left(\pi_{\alpha}(\xi)\right)_{\alpha \in I}$ are pairwise isomorphic.

Fix $\alpha_{1}<\cdots<\alpha_{m} \in I$. Let us define $r=\left(C_{r}, \pi_{r}\right)$ : put $C_{r}=C_{\alpha_{1}} \cup \cdots \cup C_{\alpha_{m}}$ and $\operatorname{dom}\left(\pi_{r}\right)=B_{\alpha_{1}} \cup \cdots \cup B_{\alpha_{m}}$. For each $\xi \in B$, let $\pi_{r}(\xi) \in \mathbb{Q}$ be the condition given by Lemma [3.6. such that $\pi_{r}(\xi) \leq \pi_{\alpha_{1}}(\xi), \ldots, \pi_{\alpha_{m}}(\xi)$. If $\xi \in B_{\alpha_{k}} \backslash B$, let $\pi_{r}(\xi)=\pi_{\alpha_{k}}(\xi)$.

Clearly $r \in \mathbb{R}$ and $C_{r} \leq C_{\alpha_{k}}$ by the definition, for all $1 \leq k \leq m$. Also, both for $\xi \in B$ and for $\xi \in B_{\alpha_{k}} \backslash B, \pi_{r}(\xi) \leq \pi_{\alpha_{k}}(\xi)$, so that $r \leq r_{\alpha_{k}}$ for all $1 \leq k \leq m$.

Theorem 3.13. If $C H$ holds in $V$, then $V^{\mathbb{R}} \vDash$ "there is no Banach space $X$ of density $\mathfrak{c}=\omega_{2}$ such that for every uniform Eberlein compact space $K$ of weight at most $\mathfrak{c}, C(K)$ can be isomorphically embedded into $X "$.

Proof. First note that $\mathbb{R} \Vdash \mathfrak{c}=\omega_{2}$. By the product lemma (see [11]), for any generic $G \subseteq \mathbb{R}$, the extension $V[G]$ is of the form $V\left[H_{1}\right]\left[H_{2}\right]$, where $H_{1}$ is a $\Pi$-generic over $V$ and $H_{2}$ is $\check{F} n_{<\omega}\left(\omega_{2}, 2\right)$-generic over $V\left[H_{1}\right]$. The first forcing $\Pi$ satisfies the $\omega_{2}$-c.c. by Lemma 3.12 and is $\sigma$-closed by Proposition 3.2 , so $V\left[H_{1}\right]$ has the same cardinals as in $V$ and satisfies the $\mathrm{CH}$. The second forcing is equal to $F n_{<\omega}\left(\omega_{2}, 2\right)$ since finite functions are the same in $V$ and $V\left[H_{1}\right]$. Hence $V[G]$ can be viewed as an extension of a model of $\mathrm{CH}$ by the Cohen forcing which adds $\omega_{2}$ Cohen reals. It is well-known (see [11]) that $\mathfrak{c}=\omega_{2}$ holds in such models.

We work in $V$. By contradiction, suppose that in $V^{\mathbb{R}}$ there is a Banach space which contains an isomorph of every UG Banach space of density $\omega_{2}$. Let $\dot{X}$ be an $\mathbb{R}$-name for it. Since $\mathbb{R} \Vdash|d(\dot{X})|=\mathfrak{c}=\omega_{2}$, we get that $\mathbb{R} \Vdash|\dot{X}|=\mathfrak{c}^{\omega}=\omega_{2}$, so that there is a family of $\mathbb{R}$-names $\left(\dot{v}_{\eta}\right)_{\eta<\omega_{2}}$ such that $\mathbb{R} \Vdash \dot{X}=\left\{\dot{v}_{\eta}: \eta<\omega_{2}\right\}$.

For each $\mathcal{F} \in\left[\omega_{2}\right]^{<\omega}$, let $\mathbb{A}_{\mathcal{F}} \subseteq \mathbb{R}$ be a maximal antichain in $\mathbb{R}$ such that for every $r \in \mathbb{A}_{\mathcal{F}}$,

$$
\text { either } r \Vdash\left\|\sum_{\eta \in \mathcal{F}} \dot{v}_{\eta}\right\|>1 \text { or } r \Vdash\left\|\sum_{\eta \in \mathcal{F}} \dot{v}_{\eta}\right\| \leq 1 \text {. }
$$

Since $\mathbb{R}$ is $\omega_{2}$-c.c., each $\mathbb{A}_{\mathcal{F}}$ has cardinality at most $\omega_{1}$ and for each $(C, \pi) \in \mathbb{A}_{\mathcal{F}}$, $|\operatorname{dom}(\pi)| \leq \omega$. Then, the set

$$
\bigcup\left\{\operatorname{dom}(\pi):(C, \pi) \in \mathbb{A}_{\mathcal{F}} \text { for some } \mathcal{F} \in\left[\omega_{2}\right]^{<\omega} \text { and } C \in F n_{<\omega}\left(\omega_{2}, 2\right)\right\}
$$

has cardinality at most $\omega_{2}$. Fix $\gamma_{0} \in \omega_{3}$ such that for every $\mathcal{F} \in\left[\omega_{2}\right]^{<\omega}$ and every $(C, \pi) \in \mathbb{A}_{\mathcal{F}}, \gamma_{0} \notin \operatorname{dom}(\pi)$.

In $V^{\mathbb{R}}$, let $K_{\gamma_{0}}$ be the Stone space of the c-algebra generated by the family $\left(A_{\xi, i}\left(\gamma_{0}\right)\right)_{\xi \in \omega_{2}, i \in \omega}$ and let us show that $C\left(K_{\gamma_{0}}\right)$ does not embed isomorphically into $\dot{X}$. For each $(i, \xi) \in \omega \times \omega_{2}$, let $\dot{A}_{\xi, i}$ be an $\mathbb{R}$-name for $A_{\xi, i}\left(\gamma_{0}\right)$.

Suppose there is $\dot{T}: C\left(K_{\gamma_{0}}\right) \rightarrow \dot{X}$ an isomorphic embedding and without loss of generality, assume that $\mathbb{R} \Vdash\|\dot{T}\|=1$. Let $r^{\prime} \in \mathbb{R}$ and find $r^{\prime \prime} \leq r^{\prime}$ and $m \in \omega$ such that $r^{\prime \prime} \Vdash\left\|\dot{T}^{-1}\right\|<\check{m}$.

For each $\alpha<\omega_{2}$, let $\left\{\xi_{k}(\alpha): 1 \leq k \leq m\right\} \subseteq \omega_{2} \backslash \alpha$ have cardinality $m$ and take $r_{\alpha} \in \mathbb{R}$ with $r_{\alpha} \leq r^{\prime \prime}$ and $\eta_{1}(\alpha), \ldots, \eta_{m}(\alpha) \in \omega_{2}$ such that

$$
r_{\alpha} \Vdash \forall 1 \leq k \leq m \quad \dot{T}\left(\chi_{\left[\dot{A}_{\check{\xi}_{k}(\alpha), k}\left(\gamma_{0}\right)\right]}\right)=\dot{v}_{\check{\eta}_{k}(\alpha)} .
$$

Let $r_{\alpha}=\left(C_{\alpha}, \pi_{\alpha}\right), s_{\alpha}=\left(C_{\alpha},\left.\pi_{\alpha}\right|_{\omega_{2} \backslash\left\{\gamma_{0}\right\}}\right) \in F n\left(\omega_{2}, 2\right) \times \Pi_{\omega_{2} \backslash\left\{\gamma_{0}\right\}}$ and $\pi_{\alpha}\left(\gamma_{0}\right)=$ $\left(D_{\alpha}, F_{\alpha}\right)$. Without loss of generality, by Lemma 3.3 we may assume that $\xi_{1}(\alpha), \ldots$, $\xi_{m}(\alpha) \in D_{\alpha}$. Using $\mathrm{CH}$ and the $\Delta$-system lemma for countable sets, we may assume 
that $\left(D_{\alpha}\right)_{\alpha<\omega_{2}}$ is a $\Delta$-system with a countable root $D$ and that $\xi_{1}(\alpha), \ldots, \xi_{m}(\alpha) \in$ $D_{\alpha} \backslash D$ for each $\alpha<\omega_{2}$ and that for each $\alpha<\omega_{2}$ the conditions $\pi_{\alpha}\left(\gamma_{0}\right)$ are isomorphic in the sense of Definition 3.5 .

By Proposition [3.10, $F n\left(\omega_{2}, 2\right) \times \Pi_{\omega_{3} \backslash\left\{\gamma_{0}\right\}}$ is isomorphic to $\mathbb{R}$. So, given $\alpha_{1}<$ $\cdots<\alpha_{m}$, we can apply Lemma 3.12 to $s_{\alpha_{1}}, \ldots, s_{\alpha_{m}}$ and get $s \in F n\left(\omega_{2}, 2\right) \times$ $\Pi_{\omega_{3} \backslash\left\{\gamma_{0}\right\}}$ such that $s \leq s_{\alpha_{1}}, \ldots, s_{\alpha_{m}}$.

Let $\mathcal{F}=\left\{\eta_{1}\left(\alpha_{1}\right), \ldots, \eta_{m}\left(\alpha_{m}\right)\right\}$ and since $\mathbb{A}_{\mathcal{F}}$ is a maximal antichain in $\mathbb{R}$ which is contained in $F n\left(\omega_{2}, 2\right) \times \Pi_{\omega_{3} \backslash\left\{\gamma_{0}\right\}}, \mathbb{A}_{\mathcal{F}}$ is also a maximal antichain in $F n\left(\omega_{2}, 2\right) \times$ $\Pi_{\omega_{3} \backslash\left\{\gamma_{0}\right\}}$. Then, there is $s^{\prime} \in \mathbb{A}_{\mathcal{F}}$ and $s^{\prime \prime} \in F n\left(\omega_{2}, 2\right) \times \Pi_{\omega_{3} \backslash\left\{\gamma_{0}\right\}}$ such that $s^{\prime \prime} \leq s, s^{\prime}$. Since $s^{\prime \prime} \leq s^{\prime}$ and $s^{\prime} \in \mathbb{A}_{\mathcal{F}}$, either

$$
s^{\prime \prime} \Vdash\left\|\dot{v}_{\check{\eta}_{1}\left(\alpha_{1}\right)}+\cdots+\dot{v}_{\check{\eta}_{m}\left(\alpha_{m}\right)}\right\| \leq 1
$$

or

$$
s^{\prime \prime} \Vdash\left\|\dot{v}_{\check{\eta}_{1}\left(\alpha_{1}\right)}+\cdots+\dot{v}_{\check{\eta}_{m}\left(\alpha_{m}\right)}\right\|>1 .
$$

Case 1. $s^{\prime \prime}$ forces that $\left\|\dot{v}_{\check{\eta}_{1}\left(\alpha_{1}\right)}+\cdots+\dot{v}_{\check{\eta}_{m}\left(\alpha_{m}\right)}\right\|>1$.

In this case, let $q \in \mathbb{Q}$ be such that $q \leq \pi_{\alpha_{1}}\left(\gamma_{0}\right), \ldots, \pi_{\alpha_{m}}\left(\gamma_{0}\right)$ obtained by Lemma 3.6 and let $r=\left(s^{\prime \prime}, q\right) \in \mathbb{R}$. Then, $r \leq s^{\prime \prime}, r \leq r_{\alpha_{1}}, \ldots, r_{\alpha_{m}}$ and $r \leq q \leq$ $\pi_{\alpha_{1}}\left(\gamma_{0}\right), \ldots, \pi_{\alpha_{m}}\left(\gamma_{0}\right)$. So,

$$
r \Vdash \forall 1 \leq k<k^{\prime} \leq m \quad \dot{A}_{\check{\xi}_{k}\left(\alpha_{k}\right), i_{k}}\left(\gamma_{0}\right) \cap \dot{A}_{\check{\xi}_{k^{\prime}}\left(\alpha_{k^{\prime}}\right), i_{k^{\prime}}}\left(\gamma_{0}\right)=\emptyset
$$

so that

Since $r \leq r_{\alpha_{1}}, \ldots, r_{\alpha_{m}}$,

$$
r \Vdash\left\|\chi_{\left[\dot{A}_{\check{\xi}_{1}\left(\alpha_{1}\right), i_{1}}\left(\gamma_{0}\right)\right]}+\cdots+\chi_{\left[\dot{A}_{\check{\xi}_{m}\left(\alpha_{m}\right), i_{m}}\left(\gamma_{0}\right)\right]}\right\|=1 .
$$

$$
r \Vdash \forall 1 \leq k \leq m \quad \dot{T}\left(\chi_{\left[\dot{A}_{\check{\xi}_{k}\left(\alpha_{k}\right), i_{k}}\left(\gamma_{0}\right)\right]}\right)=\dot{v}_{\check{\eta}_{k}\left(\alpha_{k}\right)},
$$

so that, using the fact that $\mathbb{R} \Vdash\|\dot{T}\|=1$, we get that

$$
r \Vdash\left\|\dot{v}_{\check{\eta}_{1}\left(\alpha_{1}\right)}+\cdots+\dot{v}_{\check{\eta}_{m}\left(\alpha_{m}\right)}\right\| \leq\left\|\chi_{\left[\dot{A}_{\check{\xi}_{1}\left(\alpha_{1}\right), i_{1}}\left(\gamma_{0}\right)\right]}+\cdots+\chi_{\left[\dot{A}_{\check{\xi}_{m}\left(\alpha_{m}\right), i_{m}}\left(\gamma_{0}\right)\right]}\right\|=1,
$$

contradicting the fact that $r \leq s^{\prime \prime}$ and

$$
s^{\prime \prime} \Vdash\left\|\dot{v}_{\check{\eta}_{1}\left(\alpha_{1}\right)}+\cdots+\dot{v}_{\check{\eta}_{m}\left(\alpha_{m}\right)}\right\|>1 .
$$

Case 2. $s^{\prime \prime}$ forces that $\left\|\dot{v}_{\check{\eta}_{1}\left(\alpha_{1}\right)}+\cdots+\dot{v}_{\check{\eta}_{m}\left(\alpha_{m}\right)}\right\| \leq 1$.

In this case, let $q \in \mathbb{Q}$ be such that $q \leq \pi_{\alpha_{1}}\left(\gamma_{0}\right), \ldots, \pi_{\alpha_{m}}\left(\gamma_{0}\right)$ obtained by Lemma 3.8 and let $r=\left(s^{\prime \prime}, q\right) \in \mathbb{R}$. Then, $r \leq s^{\prime \prime}, r \leq r_{\alpha_{1}}, \ldots, r_{\alpha_{m}}$ and $r \leq q \leq$ $\pi_{\alpha_{1}}\left(\gamma_{0}\right), \ldots, \pi_{\alpha_{m}}\left(\gamma_{0}\right)$. So,

$$
r \Vdash \dot{A}_{\check{\xi}_{1}\left(\alpha_{1}\right), i_{1}}\left(\gamma_{0}\right) \cap \cdots \cap \dot{A}_{\check{\xi}_{m}\left(\alpha_{m}\right), i_{m}}\left(\gamma_{0}\right) \neq \emptyset
$$

so that

$$
r \Vdash\left\|\chi_{\left[\dot{A}_{\check{\xi}_{1}\left(\alpha_{1}\right), i_{1}}\left(\gamma_{0}\right)\right]}+\cdots+\chi_{\left[\dot{A}_{\check{\xi}_{m}\left(\alpha_{m}\right), i_{m}}\left(\gamma_{0}\right)\right]}\right\|=m .
$$

Since $r \leq r_{\alpha_{1}}, \ldots, r_{\alpha_{m}}$,

$$
r \Vdash \forall 1 \leq k \leq m \quad \dot{T}\left(\chi_{\left[\dot{A}_{\check{\xi}_{k}\left(\alpha_{k}\right), i_{k}}\left(\gamma_{0}\right)\right]}\right)=\dot{v}_{\check{\eta}_{k}\left(\alpha_{k}\right)},
$$

so that, using the fact that $\mathbb{R} \Vdash\left\|\dot{T}^{-1}\right\|<m$ and that $r \leq s^{\prime \prime}$, we get that

$$
r \Vdash\left\|\chi_{\left[\dot{A}_{\check{\xi}_{1}\left(\alpha_{1}\right), i_{1}}\right]}+\cdots+\chi_{\left[\dot{A}_{\check{\xi}_{m}\left(\alpha_{m}\right), i_{m}}\right]}\right\| \leq\left\|\dot{T}^{-1}\right\| \cdot\left\|\dot{v}_{\check{\eta}_{1}\left(\alpha_{1}\right)}+\cdots+\dot{v}_{\check{\eta}_{m}\left(\alpha_{m}\right)}\right\|<m,
$$

which contradicts our assumption. 
Since the condition $r^{\prime} \in \mathbb{R}$ was arbitrary, we showed that a dense subset of $\mathbb{R}$

forces the nonexistence of the embedding $\dot{T}$; hence it does not exist in $V[G]$.

\section{REFERENCES}

1. D. Amir and J. Lindenstrauss, The structure of weakly compact sets in Banach spaces. Ann. of Math. (2) 88 (1968) 35-46. MR.0228983 (37:4562)

2. S. Argyros and Y. Benyamini, Universal WCG Banach spaces and universal Eberlein compacts. Israel J. Math. 58 (1987), no. 3, 305-320. MR917361 (89d:46016)

3. M. Bell, Universal uniform Eberlein compact spaces. Proc. Amer. Math. Soc. 128 (2000), no. 7, 2191-2197. MR:1676311 (2000m:54019)

4. M. Bell and W. Marciszewski, Universal spaces for classes of scattered Eberlein compact spaces. J. Symbolic Logic 71 (2006), no. 3, 1073-1080. MR2251557 (2007g:46012)

5. J. Clarkson, Uniformly convex spaces, Trans. Amer. Math. Soc. 40 (1936), no. 3, 396-414. MR 1501880

6. Y. Benyamini, M. Rudin and M. Wage, Continuous images of weakly compact subsets of Banach spaces. Pacific J. Math. 70 (1977), no. 2, 309-324. MR0625889 (58:30065)

7. C. Brech and P. Koszmider, On universal Banach spaces of density continuum. To appear in Israel J. Math.

8. M. Džamonja and L. Soukup, Some Notes, circulated notes, 2010.

9. M. Fabian, P. Habala, P. Hájek, V. Montesinos Santalucia, J. Pelant, and V. Zizler, Functional analysis and infinite-dimensional geometry, CMS Books in Mathematics/Ouvrages de Mathématiques de la SMC, 8, Springer-Verlag, New York, 2001. MR1831176 (2002f:46001)

10. M. Fabian, G. Godefroy, and V. Zizler, The structure of uniformly Gateaux smooth Banach spaces. Israel J. Math. 124 (2001), 243-252. MR.1856517 (2002g:46015)

11. K. Kunen, Set Theory. An introduction to independence proofs, Studies in Logic and the Foundations of Mathematics, 102, North-Holland, Amsterdam, 1980. MR597342 (82f:03001)

12. Z. Semadeni, Banach spaces of continuous functions, Monografie Matematyczne, Tom 55, Państwowe Wydawnictwo Naukowe, Warsaw, 1971. MR0296671 (45:5730)

13. S. Todorcevic, Embedding function spaces into $\ell_{\infty} / c_{0}$, J. Math. Anal. Appl. 384 (2011), no. 2, 246-251. MR2825178

Departamento de Matemática, Instituto de Matemática e Estatística, Universidade de SÃo Paulo, Caixa Postal 66281, 05314-970, São Paulo, Brazil

E-mail address: christina.brech@gmail.com

Institute of Mathematics, Technical University of Łódź, Ul. Wólczańska 215, 90-924 ŁÓDŹ, POLAND

Current address: Institute of Mathematics, Polish Academy of Sciences, ul. Śniadeckich 8, 00-956 Warszawa, Poland

E-mail address: piotr.math@gmail.com 